\title{
Increased Runx2 expression associated with enhanced Wnt signaling in PDLLA internal fixation for fracture treatment
}

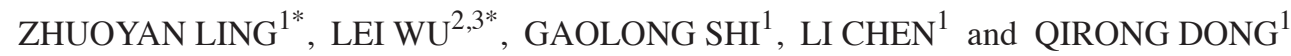 \\ ${ }^{1}$ Department of Orthopedics, The Second Affiliated Hospital of Soochow University, Suzhou, Jiangsu 215006; \\ ${ }^{2}$ Centers for Disease Control and Prevention of Suzhou Industrial Park, Suzhou, Jiangsu 215021; \\ ${ }^{3}$ School of Biology and Basic Medical Sciences, Soochow University, Suzhou, Jiangsu 215123, P.R. China
}

Received October 14, 2015; Accepted November 10, 2016

DOI: 10.3892/etm.2017.4216

\begin{abstract}
Poly-D-L lactide (PDLLA) biodegradable implants to heal fractures are widely applied in orthopedic surgeries. However, whether the process of fracture healing is regulated differently when PDLLA is used compared with traditional metal materials remains unclear. Runt-related transcription factor 2 (Runx2) and canonical Wnt signaling are essential and may interact reciprocally in the regulation of osteogenesis during bone repair. In the present study, a rat femoral open osteotomy model was used to compare the curative efficacy of a PDLLA rod and Kirschner wire under intramedullary fixation for fracture treatment. The dynamic expression of Runx2 and key components of the canonical Wnt signaling in callus tissue during fracture healing was also investigated. The results of the current study indicate that at weeks 4 and 6 following fixation, the callus bone structural parameters of microCT were significantly improved by PDLLA rod compared to that of Kirschner wire. In addition, at weeks 4 and 6 after fixation, the protein and mRNA expression of Runx 2 and the positive regulators of canonical Wnt signaling, such as Wnts and $\beta$-catenin, were significantly increased. However, the protein and mRNA expression levels of the negative regulators of canonical Wnt signaling, such as glycogen synthase kinase-3 $\beta$, were significantly decreased in callus tissue when treated with PDLLA rod compared with Kirschner wire. Collectively, these data indicate that compared to the traditional metal material, using PDLLA internal fixation for fracture treatment may further improve bone formation, which is associated with the increased expression of Runx 2 and the enhancement of canonical Wnt signaling.
\end{abstract}

Correspondence to: Professor Qirong Dong, Department of Orthopedics, The Second Affiliated Hospital of Soochow University, 1055 San Xiang Road, Suzhou, Jiangsu 215006, P.R. China E-mail: drq0512@126.com

\section{*Contributed equally}

Key words: Poly-D-L lactide material, metal material, fracture healing, runt-related transcription factor 2, canonical Wnt signaling

\section{Introduction}

Since the use of biodegradable implants to heal fractures by Rokkanen et al in 1985, the method of using biodegradable materials has been widely applied in orthopedic surgeries (1-3). Biodegradable materials exhibit various advantages in the treatment of fractures compared with the use of traditional metal implants, including the elimination of implant removal, reduction of the 'stress shielding' effect, improvement of biocompatibility, reduction of radiological artifacts and utilization of magnetic resonance imaging assessment following surgery $(4,5)$. Poly-D-L lactide (PDLLA) is a material with an intermediate degradation time (PDLLA begins to degrade at $\sim 12$ weeks) and may be completely replaced by bone tissue following surgery, thus, it is considered to be one of the most effective biodegradable materials for the treatment of fractures (6-8). However, with the exception of clinical outcomes, the differences in the biological processes following the use of PDLLA and metal fixation to treat a fracture remain unknown.

The fracture healing process has been widely accepted to comprise a series of overlapping phases; these include inflammation, repair and remodeling events (9). This multistage repair process involves a variety of complex and well-orchestrated cellular and molecular processes, and should be considered as a specialized post-natal recapitulation of embryological skeletal development $(9,10)$. Runt-related transcription factor 2 (Runx2) is one member of the Runx family that belongs to a small transcription factor family sharing a common runt domain (11). Previous studies indicated that Runx2 was a crucial transcriptional regulator for skeletal development and served essential roles in biological events including osteoblast differentiation, intramembranous, endochondral ossification and in the process of fracture healing, vascular invasion (12-14). However, whether the expression of Runx2 during fracture healing may be altered by the use of PDLLA compared to that of a traditional metal material for fracture treatment remains unknown.

A previous study demonstrated that the canonical Wnt signaling served essentials roles in regulating bone formation during the fracture repair process (15). In the canonical Wnt signaling pathway, the Wnts proteins, a family of secreted glycoproteins, bind to the membrane receptor Frizzled (Fz) leading to downstream sub-cellular events. The disheveled 
protein, along with other co-receptors, is activated to inhibit the activity of glycogen synthase kinase- $3 \beta$ (GSK-3 $\beta$ ). $\beta$-catenin is able to avoid the degradation of the protein ubiquitinylation system and translocate into the nucleus in order to associate with transcription factors, including $\mathrm{T}$ cell factor-1 (TCF-1), in order to regulate the transcriptional activities of relevant genes (16). A number of studies have indicated that the expression of certain Wnt proteins, including Wnt4, 5b, 10b, 11, and 13, and $\mathrm{Fz}$ receptors such as Fz1, 2, 4, and 5, are increased during the fracture healing process $(17,18)$. The expression of Runx 2 may also be regulated by the activity of canonical Wnt signaling through its binding with $\beta$-catenin/TCF-1 in certain promoter regions of the runx 2 gene (19). However, whether and to what extent the activity of the canonical Wnt signaling in the bone repair process may be changed by PDLLA, compared to that of traditional metal material for fracture treatment, has not yet been determined.

Overall, the present study established an open osteotomy model of Sprague-Dawley rats in order to determine whether and to what extent the expression of Runx 2 and the activity of canonical Wnt signaling during bone repair process may be differentially regulated. The use of traditional metal material (Kirschner wire) and PDLLA intramedullary rod under internal fixation for fracture treatment was assessed. The results of the present study indicate that compared to the traditional metal material, the curative efficacy of PDLLA internal fixation for fracture treatment is improved, mediated by the increased Runx 2 expression associated with the enhanced activity of canonical Wnt signaling.

\section{Materials and methods}

Experimental animals. A total of 36 male Sprague-Dawley rats (six months old) weighing 280-300 g were supplied by the Laboratory Animal Research Center of Soochow University (Suzhou, China). They were maintained under standard conditions for at least one week, and individually housed in plastic cages in an animal room in a temperature-controlled environment of $22-25^{\circ} \mathrm{C}$ and relative humidity of $40-70 \%$ with a 12-h light/dark cycle and ad libitum access to commercial pellets and water. Animals were randomly assigned into control and PDLLA groups ( $n=18$ per group). Following the transverse osteotomy at the mid-shaft of right femur, the traumatic fracture was stabilized intramedullary by the Kirschner wire and PDLLA rod in control and PDLLA groups, respectively. All procedures were approved by the Institutional Animal Care Service (Second Affiliated Hospital of Soochow University).

Surgical procedure of femoral open osteotomy model. The osteotomy was performed using a circular saw with a diameter of $1.6 \mathrm{~cm}$ and a thickness of $0.1 \mathrm{~mm}$ at mid-shaft of the right femur, under the intraperitoneal injection of $50 \mathrm{mg} / \mathrm{kg}$ sodium pentobarbital (Sinopharm Chemical Reagent, Co., Ltd., Shanghai, China). The fracture was stabilized by intramedullary insertion of a sterilized $1 \mathrm{~mm}$ diameter Kirschner wire (Nantong Healthcare Medical Instrument, Co., Ltd., Nantong, China) in the control group and a sterilized $1 \mathrm{~mm}$ diameter PDLLA rod (Nantong Healthcare Medical Instrument, Co., Ltd.) in the PDLLA group. At weeks 2, 4 and 6 post-surgery,
$50 \mathrm{mg} / \mathrm{kg}$ sodium pentobarbital was used to anaesthetize 6 rats from each group prior to sacrifice via cervical dislocation.

Alkaline phosphatase and osteoprotegerin serum levels. Blood samples ( $0.5 \mathrm{ml}$ each) were obtained using an intravenous tube implanted in the femoral vein following 2, 4 and 6 weeks and stored at $-20^{\circ} \mathrm{C}$ until analysis. The concentrations of alkaline phosphatase (ALP) and osteoprotegrin (OPG) were detected in the serum using commercially available ELISA kits (C506082-0096, and C506509-0048; Sangon Biotech, Co., Ltd., Shanghai, China).

MicroCT scanning and assessment of bone quality parameters. At weeks 2, 4 and 6 post-surgery, six rats randomly selected from each group were anesthetized with intraperitoneal injections of sodium pentobarbital (Sinopharm Chemical Reagent, Co., Ltd., $50 \mathrm{mg} / \mathrm{kg}$ ) prior to micro-CT scanning. The femora were scanned by a desktop micro-CT system (IVIS Quantum FX; Cold Spring Biotech Corporation, Shanghai, China) with the resolution of $47 \mu \mathrm{m}$ and scanning time of $15 \mathrm{~min}$ each. Values of bone volume fraction (BV/TV), bone surface density (BS/BV), trabecular thickness (TB.TH), trabecular number (TB.N), and trabecular separation (TB.SP) were determined in each group under the certain regions of interest. The scan area was centered through the fracture line.

Western blot analysis. Protein samples were routinely extracted from callus tissue using a Total Protein Extraction kit according to the manufacturer's protocol (310003; BestBio Biotechnology Co., Ltd., Shanghai, China) 2, 4 and 6 weeks following surgery, as described previously (20). Samples of protein $(80 \mu \mathrm{g})$ were loaded in each lane for electrophoresis in a $4 \%$ SDS-PAGE gel ( $0.1 \%$ SDS and $10 \%$ polyacrylamide) and transferred to immobilon $\mathrm{P}$ polyvinyldifluoride membranes (EMD Millipore, Billerica, MA, USA). Each membrane was blocked with Tris-buffered PBS containing 5\% bovine serum albumin (Chinese Academy of Sciences, Shanghai, China) plus $0.1 \%(\mathrm{v} / \mathrm{v})$ Tween (TBS-T) on a gentle shaker for $1 \mathrm{~h}$ at room temperature, followed by incubation at $4{ }^{\circ} \mathrm{C}$ overnight with rabbit polyclonal antibodies (Santa Cruz Biotechnology, Inc. Santa Cruz, CA, USA) against Runx2 (sc-10758, 1:1,000), hypoxia inducible factor 2A (HIF2A; sc-28706, 1:1,000), Wnt4 (sc-13962, 1:1,000), Wnt10b (sc-25524, 1:1,000), GSK-3 $\beta$ (sc-9166, 1:1,000), $\beta$-catenin (sc-7199, 1:1,000) and TCF-1 (sc-13025, 1:1,000). Membranes were then washed three times for $10 \mathrm{~min}$ in TBS-T solution and incubated at room temperature with the secondary horseradish peroxidase-conjugated goat anti-rabbit antibody (1:5,000; A0208; Beyotime Institute of Biotechnology, Haimen, China) for $2 \mathrm{~h}$. Proteins were then visualized using enhanced chemiluminescence detection reagents (ECL reagent RPN2232, GE Healthcare Life Sciences, Chalfront, UK) and exposed to X-ray film according to the manufacture's protocol. The $\beta$-actin protein (primary antibody from EMD Millipore, working concentration: $1: 1,000)$ was blotted in the same membrane as an internal control to normalize the relative density. Results were quantified and analyzed using a Kodak electrophoresis documentation and analysis system, and Kodak ID image analysis software (Kodak, Rochester, NY, USA). Three replicates were performed per tissue sample. 
Table I. Comparison of microCT analysis of fracture calluses between PDLLA group and control group across time-points.

\begin{tabular}{lccc}
\hline microCT parameters & Weeks after fixation & Con & PDLLA \\
\hline BV/TV, $\%$ & 2 & $2.469 \pm 0.14$ & $2.435 \pm 0.11$ \\
& 4 & $3.188 \pm 0.16$ & $3.765 \pm 0.13^{\mathrm{a}}$ \\
& 6 & $5.132 \pm 0.18$ & $6.123 \pm 0.13^{\mathrm{a}}$ \\
$\mathrm{BS} / \mathrm{BV}, 1 / \mathrm{m}$ & 2 & $1.887 \pm 0.14$ & $1.869 \pm 0.12$ \\
& 4 & $2.025 \pm 0.11$ & $2.183 \pm 0.12^{\mathrm{a}}$ \\
$\mathrm{BV} / \mathrm{TV}, \%$ & 6 & $3.102 \pm 0.12$ & $3.244 \pm 0.16^{\mathrm{a}}$ \\
& 2 & $0.0740 \pm 0.0011$ & $0.0738 \pm 0.0014$ \\
$\mathrm{BS} / \mathrm{BV}, 1 / \mathrm{m}$ & 4 & $0.0751 \pm 0.0013$ & $0.0768 \pm 0.0011^{\mathrm{a}}$ \\
& 6 & $0.0831 \pm 0.0012$ & $0.0857 \pm 0.0015^{\mathrm{a}}$ \\
$\mathrm{BV} / \mathrm{TV}, \%$ & 2 & $0.334 \pm 0.031$ & $0.333 \pm 0.043$ \\
& 4 & $0.491 \pm 0.034$ & $0.522 \pm 0.062^{\mathrm{a}}$ \\
& 6 & $0.654 \pm 0.051$ & $0.709 \pm 0.061^{\mathrm{a}}$ \\
& 2 & $1.106 \pm 0.0051$ & $1.108 \pm 0.0044$ \\
& 4 & $1.083 \pm 0.0072$ & $1.073 \pm 0.0035^{\mathrm{a}}$ \\
\hline
\end{tabular}

Data are presented as mean \pm standard deviation. ${ }^{\mathrm{a}} \mathrm{P}<0.05$. Con group, control group; PDLLA, Poly-D-L lactide treated group; BV/TV, bone volume/tissue volume; $\mathrm{BS} / \mathrm{BV}$, bone volume/bone volume.

Reverse transcription-quantitative polymerase chain reaction $(R T$ - $q P C R)$. At weeks 2, 4 and 6 post-surgery, total tissue RNA was routinely isolated from the callus tissue using Eastep Super Total RNA Extraction kit (Promega Corporation, Madison, WI, USA) according to the manufacturer's protocol. Reverse transcription and first strand cDNA synthesis was performed using M-MLV Reverse Transcriptase according to the manufacture's protocol (Invitrogen; Thermo Fisher Scientific Inc., Waltham, MA, USA). RT-qPCR analysis was completed for Runx2, HIF2A, Wnt4, Wnt10b, GSK-3 $\beta$, $\beta$-catenin and TCF-1 gene expression in callus tissue. The GAPDH gene was used as an internal control and serial dilutions of the positive control (the synthesized tissue cDNA from rat bone; RD-107, Zyagen, San Diego, CA, USA) were performed on each plate to create a standard curve. The primer sequences (Sangon Biotech, Co., Ltd.) are as follows: For rat Runx2 are forward, 5'-CACAAGTGCGGTGCAAACTT-3' and reverse, 5'-AAT GACTCGGTTGGTCTCGG-3'; for rat HIF2A are forward 5'-AACCTTAAGTCGGCCACCTG-3' and reverse, 5'-TTG CTGTCCAAGGGGATGTC-3'; for rat Wnt4 are forward, 5'-TACGGATGAGGACCTGGTGT-3' and reverse, 5'-GAC GTCTTGTTGCAAGTGCG-3'; for rat Wnt10b are forward, 5'-CCGTGAGTTAGGTCGAGCAG-3' and reverse, 5'-GTG GGGAAACTGTGTGGAGT-3'; for rat GSK-3 $\beta$ are forward, 5'-CGAACTCCACCAGAGGCAAT-3' and reverse, 5'-GAG TTGGAGGCTGATGCAGA-3'; for rat $\beta$-catenin are forward, 5'-CTGCTGATCTCGGACTGGAC-3' and reverse, 5'-GGC AGCCCATCAACTGGATA-3'; for rat TCF-1 are forward, 5'-CAGAATCCACAGATACAGCA-3' and reverse, 5'-CAG CCTTTGAAATCTTCATC-3'; for rat GAPDH are forward, 5'-GGTGGTCTCCACGGACTTTA-3' and reverse, 5'CAA GGAGGGGCCTTTATTTC3'. RT-qPCR (iCycler iQ ${ }^{\mathrm{TM}}$ Real-time PCR detection system; Bio-Rad Laboratories, Inc., Hercules, CA, USA) was performed using $25 \mu 1$ of each sample, in 96-well plates (Takara Bio, Inc., Otsu, Japan) under the following protocol: $42^{\circ} \mathrm{C}$ for $30 \mathrm{~min}, 95^{\circ} \mathrm{C}$ for $15 \mathrm{~min}$ followed by 40 cycles of $95^{\circ} \mathrm{C}$ for $20 \mathrm{sec}, 56^{\circ} \mathrm{C}$ for $1 \mathrm{~min}$ and $72^{\circ} \mathrm{C}$ for $20 \mathrm{sec}$. The amount of target gene was normalized to the GAPDH reference to obtain the relative threshold cycle $(\Delta \Delta \mathrm{Cq})$, then $2^{-\Delta \Delta \mathrm{Cq}}$ was calculated to determine the relative abundance for target gene expression between control and PDLLA groups (21).

Statistical analysis. All data were expressed as mean \pm standard deviation. With regard to the different mRNA level of PDLLA and control groups, statistical significance was accepted $(\mathrm{P}<0.05)$ when the ratio of $2^{-\Delta \Delta \mathrm{Cq}}$ exceeded 1.7 . The two-way analysis of variance and independent $t$-test, where appropriate, was used to evaluate the differences between PDLLA and control groups. A statistically significant difference was accepted if $\mathrm{P}<0.05$. SPSS statistical software (version 18; SPSS, Inc., Chicago, IL, USA) was used for all statistical analyses.

\section{Results}

MicroCT analysis offracture calluses. MicroCT analysis indicated that, compared with the control, the values of trabeculae quality of the traumatic femur including BV/TV, BS/TV, TB.TH, TB.N and TB.SP were significantly improved in the PDLLA group, 4 and 6 weeks following the intramedullary fixation for femoral osteotomy $(\mathrm{P}<0.05)$. However, at week 2 there was no significant difference in the values of trabeculae growth between the PDLLA group compared with the control $(\mathrm{P}>0.05$; Table I).

Serum values of $A L P$ and $O P G$. In order to further evaluate the differences in osteogenesis between the PDLLA and 

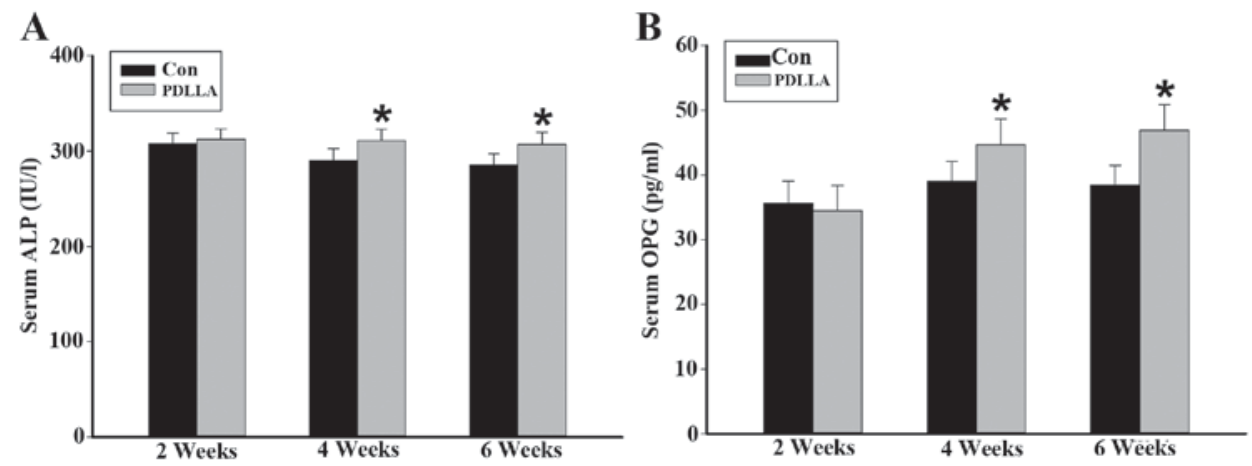

Figure 1. ALP and OPG serum levels in rats 2, 4 and 6 weeks post-surgery. (A) Serum level of ALP and (B) OPG at weeks 2, 4 and 6 post-surgery, under PDLLA intramedullary fixation (PDLLA group) compared to that of Kirschner wire (control group). Values are mean \pm standard deviation, "P<0.05. Con, control group; PDLLA, Poly-D-L lactide treated group; ALP, alkaline phosphatase; OPG, osteoprotegerin.

control group, the circulatory serum levels of ALP and OPG were detected as they are widely considered to be markers of bone formation. The ELISA assay indicated that weeks 4 and 6 following the intramedullary fixation for femoral osteotomy, the serum levels of ALP and OPG were significantly increased in the PDLLA group compared with that of the control $(\mathrm{P}<0.05)$. However, results from week 2 demonstrate that the serum levels of ALP and OPG are not significantly different in the PDLLA group compared to that of control ( $\mathrm{P}>0.05$; Fig. 1).

Protein expression of Runx2, HIF $2 A$, Wnts, GSK-3 $\beta$, $\beta$-catenin and TCF-1 in callus tissue. To compare the differences of cellular and molecular events involved in the process of fracture healing between the PDLLA and control groups, runx2, canonical Wnt signaling and associated regulators were dynamically examined. Western blot analysis demonstrated that 4 and 6 weeks following the intramedullary fixation for femoral osteotomy, the protein expression levels of Runx2, HIF2A, Wnts and $\beta$-catenin in callus tissue were significantly increased in the PDLLA group compared with the control $(\mathrm{P}<0.05$; Fig. 2A-E). Furthermore, the level of GSK-3 $\beta$ protein expression in callus tissue, at week 4 and 6 , was significantly decreased in the PDLLA group compared to that of control $(\mathrm{P}<0.05$; Fig. 2F). However, at week 2 following the intramedullary fixation, the level of protein expression for Runx2, HIF2A, Wnts, $\beta$-catenin and GSK-3 $\beta$ exhibited no significant differences in the PDLLA group compared with that of control (P>0.05; Fig. 2A-F). With respect to TCF-1 at weeks 2, 4 and 6 following the intramedullary fixation for femoral osteotomy, the protein expression levels exhibited no significant differences in the PDLLA group compared with that of control ( $P>0.05$; Fig. $2 \mathrm{G})$.

Runx2, HIF2A, Wnts, GSK-3 $\beta, \beta$-catenin and TCF-1 mRNA in callus tissue. It was demonstrated following RT-qPCR that, similar to the of protein expression, 4 and 6 weeks following the intramedullary fixation for femoral osteotomy, the expression of Runx2, HIF2A, Wnts and $\beta$-catenin mRNA in callus tissue were significantly increased in the PDLLA group compared with that of the control $(\mathrm{P}<0.05$; Fig. 3A-E). The mRNA expression levels of GSK-3 $\beta$ in callus tissue, at weeks 4 and 6 were significantly decreased in the PDLLA group compared with the control $(\mathrm{P}<0.05)$. However, 2 weeks after the intramedullary fixation, the level of mRNA expression observed for Runx2, HIF2A, Wnts, $\beta$-catenin and GSK-3 $\beta$ exhibited no significant difference in the PDLLA group compared with the control (Fig. 3A-F; P>0.05). With respect to TCF-1, at weeks 2,4 and 6 following the intramedullary fixation for femoral osteotomy, the mRNA expression levels exhibited no significant differences in PDLLA group compared to that of control (Fig. 3G; P>0.05).

\section{Discussion}

In the present study, the fracture bone repair under intramedullary fixation was improved following the use of PDLLA instead of traditional metal material. Furthermore, compared with the traditional metal material, the expression of Runx 2 in callus tissue, which is closely associated with the further activation of canonical Wnt signaling, was significantly increased under PDLLA intramedullary fixation.

With the development and improvement of pixel resolution and the mathematical combination with finite element analysis, microCT has been widely applied in assessing the biomechanical features and qualities, including bone mineral density and Young's modulus, of cortical and trabecular bone (22). In the present study, microCT-scanning results indicated that following 4 and 6 weeks post-surgery, the fracture repair indices, including BV/TV, BS/BV, TB.TH, TB.N and TB.SP, were significantly improved in the PDLLA group compared with the control group. ALK and OPG were expressed in the bone tissue, specifically in osteoblastic lineages and their serum concentrations were considered as the markers for the evaluation of osteogenesis during development and bone formation throughout fracture healing $(23,24)$. The results of the current study indicate that at weeks 4 and 6 following intramedullary fixations for osteotomy, the serum levels of ALK and OPG were significantly increased in the PDLLA group, compared with the control. Thus, the current study indicates that compared to the traditional metal material, the osteogenesis in the process of fracture healing was increasingly activated and therefore further improved the curative efficacy of PDLLA intramedullary fixation for fracture treatment.

It has been indicated that the Runx 2 is extensively expressed in the callus tissue during the process of fracture 

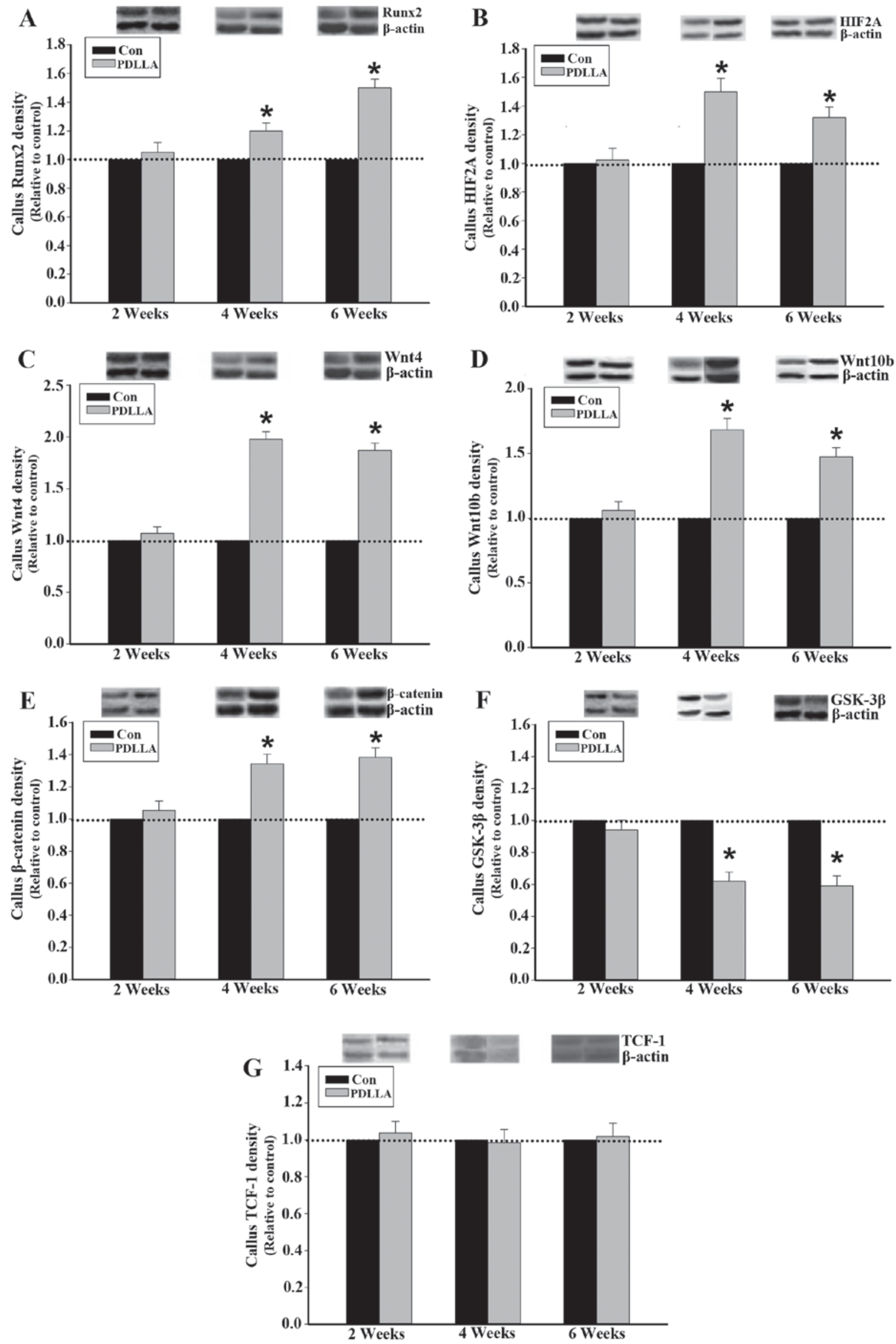

Figure 2. Protein expression in callus tissue of control and PDLLA treated rats 2, 4 and 6 weeks post-surgery. Expression level of (A) Runx2, (B) HIF2A, (C) Wnt4, (D) Wnt10b, (E) GSK-3ß, (F) $\beta$-catenin and (G) TCF-1 under PDLLA intramedullary fixation (PDLLA group) compared to that of Kirschner wire (control group). B-actin is a control. Values are presented as mean \pm standard deviation, ${ }^{*} \mathrm{P}<0.05$. Con, control group; PDLLA, Poly-D-L lactide treated group; Runx2, Runt-related transcription factor 2; HIF2A, hypoxia inducible factor 2A; GSK-3 $\beta$, glycogen synthase kinase-3 $\beta$; TCF-1, T cell factor-1. 
A

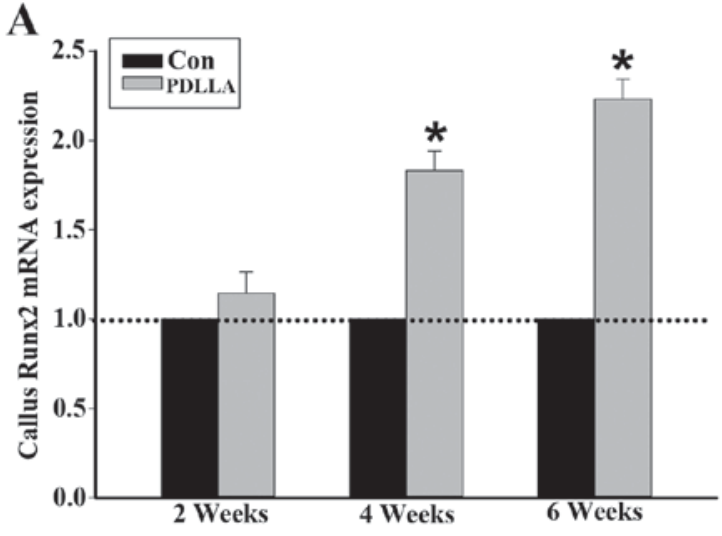

C
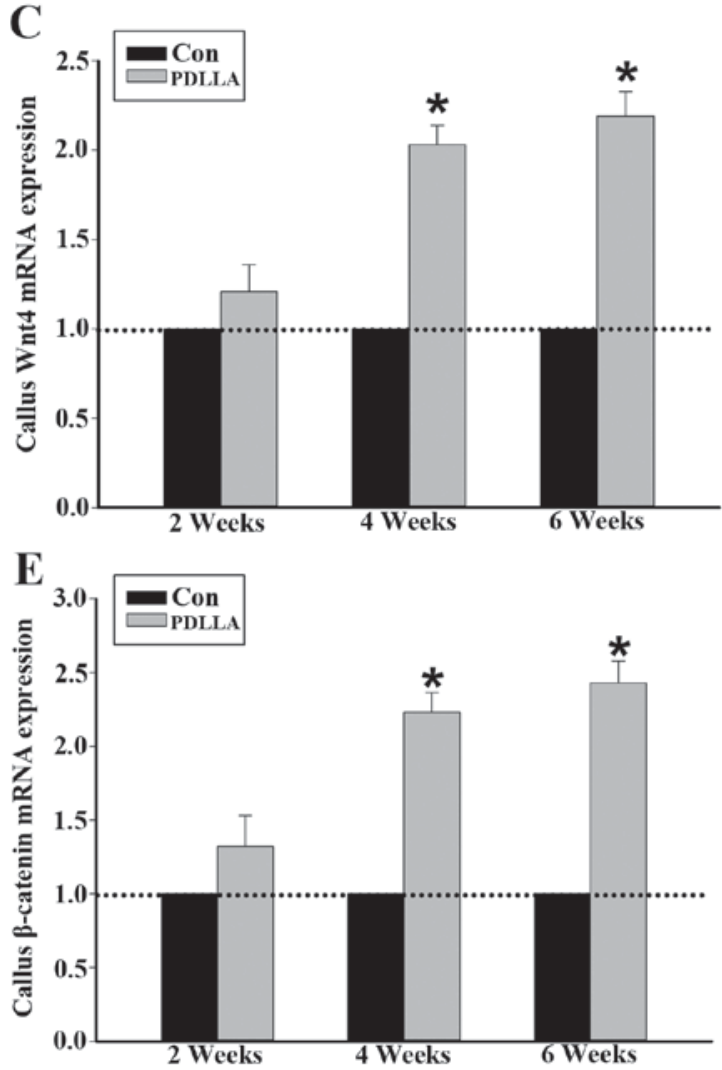

B

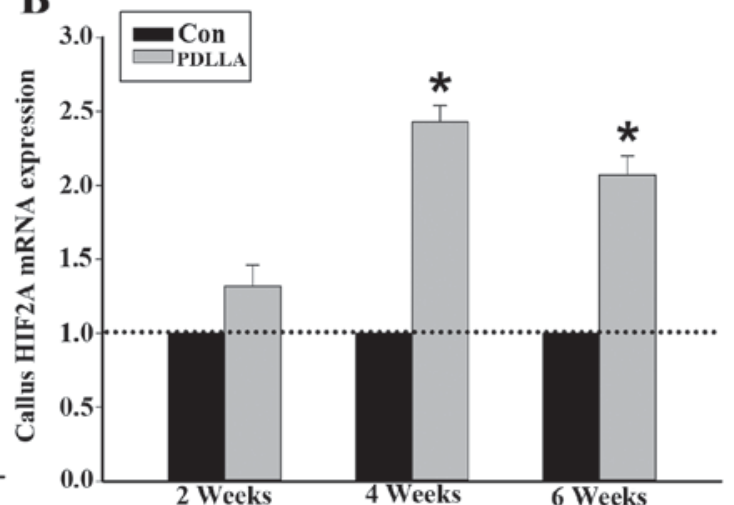

D

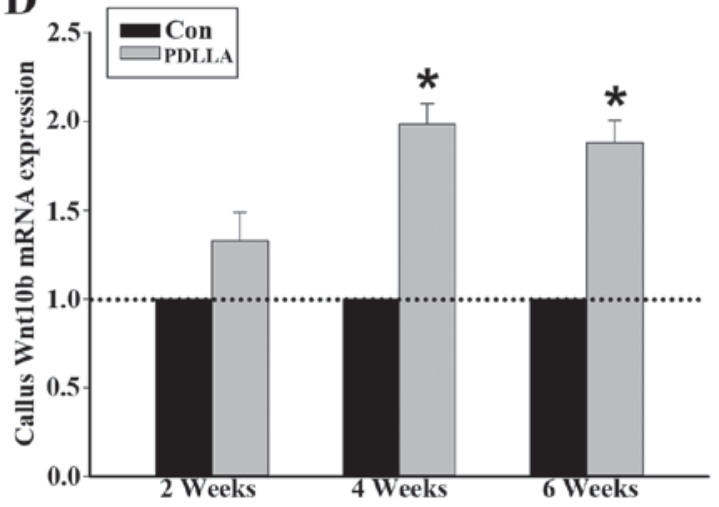

F

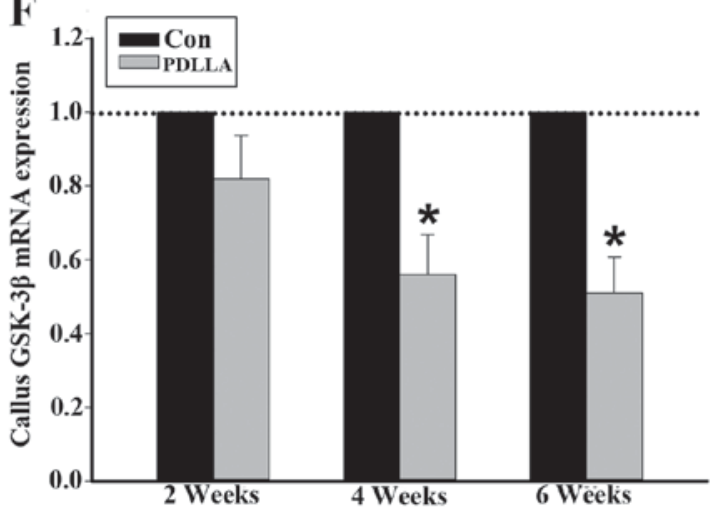

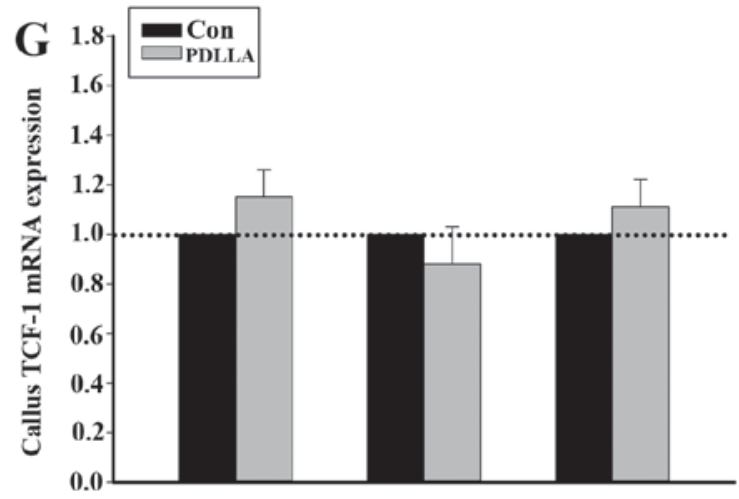

Figure 3. mRNA expression in callus tissue of control and PDLLA treated rats 2, 4 and 6 weeks post-surgery. Expression level of (A) Runx2, (B) HIF2A, (C) Wnt4, (D) Wnt10b, (E) GSK-3 $\beta$, (F) $\beta$-catenin and (G) TCF-1. B-actin is a control. Values are presented as mean \pm standard deviation, ${ }^{*} \mathrm{P}<0.05$. Con, control group; PDLLA, Poly-D-L lactide treated group; Runx2, Runt-related transcription factor 2; HIF2A, hypoxia inducible factor $2 \mathrm{~A}$; GSK-3 $\beta$, glycogen synthase kinase-3 $\beta$; TCF-1, T cell factor-1.

healing (25). In the present study, the level of mRNA and protein expression of Runx2 in the callus tissue of the PDLLA group were significantly increased, compared to that of control, at 4 and 6 weeks following intramedullary fixation for 
osteotomy. Genetic engineering studies demonstrated that the altered expression of Runx 2 may lead to abnormal and lethal bone development and ossification (26). Studies have indicated that Runx2 may serve as an essential transcriptional factor to extensively regulate the osteogenesis mediated by a number of pathways, including the differentiation and maturation of osteoblasts and osteoclasts, expression of bone matrix protein genes and angiogenesis controlled by vascular endothelial growth factor $(27,28)$. Thus, the present study proposed that the improved curative efficacy of PDLLA in the intramedullary fixation for traumatic fracture was closely associated with the increased expression of Runx 2 in callus tissue; an important regulator for osteogenesis in the process of traumatic bone repair. However, the pathways involved and the aforementioned underlying cellular and molecular mechanisms may be differentially regulated by the increased expression of Runx2, thus more studies are required.

The Runx2 gene spans $\sim 210 \mathrm{~kb}$ with its two predominant transcripts, governed by P1 and P2 promoters (29,30). Runx2 RNA, driven by the P1 promoter, is extensively stimulated and highly expressed in osteoblastic lineages during skeletal development (31). Previous results have demonstrated that the specific loss of expression of the Runx2-P1 promoter in mice leads to severe developmental defects with cleidocranial dysplasia-like symptoms (32). Thus, the activity of the P1 promoter serves a crucial role in the regulation of Runx 2 transcription in the process of osteogenesis. Previous experiments indicated that HIF2A may bind to the -155 and -75 bp region of the Runx2-P1 promoter and serve as upstream regulators for the basal transcription of the Runx 2 gene (33). In the present study, results indicated that 4 and 6 weeks following intramedullary fixation for osteotomy, the mRNA and protein expression of HIF2A in callus tissue were significantly increased in the PDLLA group compared to the control. It was therefore hypothesized that, compared to traditional metal material, the use of PDLLA for internal fixation of fractures increases the expression of HIF2A. This further promotes the transcriptional activity of Runx2 through an interaction between HIF2A and the Runx2-P1 promoter during the process of traumatic bone repair (34). Notably, various studies indicated that, from the second week following PDLLA implantation, the material began to be degraded $(35,36)$. The biodegraded monomer would then enter into the citric acid cycle to yield carbon dioxide and water (35). As a consequence, when compared to the traditional metal material, the oxygen level in the local repair tissue decreases at weeks 4 and 6 following intramedullary fixations by PDLLA. Thus, under PDLLA internal fixation for fracture treatment, the expression of HIF2A may have increased due to the relative hypoxia condition in the callus tissue. However, the concerned data supporting this hypothesis in this study is lacking and the detailed mechanism requires further study to be elaborated.

It is acknowledged that the canonical Wnt signaling may govern the lineage commitment and differentiation of progenitor cells, specifically the mesenchymal stem cells, into the chondrocytes and osteoblasts, so as to serve the critical roles in the process of osteogenesis (37). Gain- and loss-of-function mutations in canonical Wnt signaling components including Wnt ligands have elaborated the critical functions of canonical Wnt signaling during endochondral bone formation (15). The canonical Wnt signaling is initiated by the binding of Wnt proteins and its Fz receptors. Following the activation of the $\mathrm{Fz}$ receptor complex, the protein phosphorylation cascade, triggered by GSK-3 $\beta$, may be inhibited to stabilize the intracellular $\beta$-catenin levels (38). Subsequently, $\beta$-catenin is translocated to the nucleus to form a heterodimeric DNA-binding complex with TCF-1 to regulate the transcription activity of downstream genes such as connexin 43 and Runx2 (39). The current study demonstrated that at weeks 4 and 6 following intramedullary fixation for osteotomy, the mRNA and protein expression levels of $\mathrm{Wnt} 4$, Wnt10b and $\beta$-catenin were significantly increased, while mRNA and protein expression levels of GSK-3 $\beta$ were significantly decreased in the PDLLA group compared with the control. Consequently, it is suggested that, compared to the traditional metal material, the canonical Wnt signaling pathway was further activated under PDLLA internal fixation for fracture treatment. Notably, previous findings indicated that, the heterodimeric DNA-binding complex of $\beta$-catenin and TCF-1 may target the -97 and -93 bp region of the Runx2-P1 promoter, enhancing the transcription activity of Runx 2 gene (19). Although the expression of TCF-1 was not changed, the increased expression of $\beta$-catenin may, at least in part, contribute to further activation and transcriptional activity of Runx 2 gene under PDLLA intramedullary fixation compared to that of metal material. The binding sites for $\beta$-catenin, TCF-1 and HIF2A in the P1 promoter region of the Runx 2 gene overlapped, the current study hypothesized that the function of $\beta$-catenin, TCF-1 and HIF2A may synergistically involve the transcriptional regulation of Runx 2 gene.

It should be noted that at week 2 following the intramedullary fixation for osteotomy, compared with traditional metal materials, all parameters detected in the current study presented little difference following the use of PDLLA biodegradable material. A number of potential reasons for this are as follows: In one aspect, previous results have indicated that PDLLA degrades relatively slowly in the first two weeks in vivo (40). This indicated that the mechanical strength PDLLA is greater than the callus tissue and the released monomer and changes to microenvironment in local tissue are limited. As such, compared to the traditional metal material, the differences concerning stress shielding and local inflammatory effects induced by PDLLA would be negligible. Secondly, during the first two weeks following the fracture in rats, the bone repair process is still in the inflammatory and initial repair phases $(9,10)$. This indicates that the biological processes of fracture healing were in the most active period and may not be clearly altered by the relatively subtle differences between the traditional metal and PDLLA materials. Over time, the bone repair process moves into the late repair and remodeling phases and new blood vessels also become mature. Furthermore, the degradation process of PDLLA material in vivo would become more active and the differences between traditional metal and PDLLA materials, including stress shielding and local inflammatory effects, would become progressively apparent. However, further investigation is required to assess which of the aforementioned factors serve as the trigger and are therefore important for changing the pathways examined in this study.

In conclusion, the findings of the present study provide a novel insight into the differences of cellular and molecular 
pathways in the process of bone repair under the internal fixation for traumatic fracture treatment using PDLLA, a biodegradable material compared to that of traditional metal materials. Although these results were obtained from the rat model, the present study may facilitate further studies of the relevant biological mechanisms underlying PDLLA in clinical use.

\section{Acknowledgements}

The present study was supported by research grants from Suzhou Science and Education Project (no. KJXW2014010) and Suzhou Science and Technology Project (no. SYS201405).

\section{References}

1. Rokkanen P, Böstman O, Vainionpää S, Vihtonen K, Törmälä P, Laiho J, Kilpikari J and Tamminmäki M: Biodegradable implants in fracture fixation: Early results of treatment of fractures of the ankle. Lancet 1: 1422-1424, 1985.

2. Zantop T, Weimann A, Schmidtko R, Herbort M, Raschke MJ and Petersen W: Graft laceration and pullout strength of soft-tissue anterior cruciate ligament reconstruction: In vitro study comparing titanium, poly-d,l-lactide, and poly-d,l-lactide-tricalcium phosphate screws. Arthroscopy 22: 1204-1210, 2006.

3. Mukherjee DP and Pietrzak WS: Bioabsorbable fixation: Scientific, technical, and clinical concepts. J Craniofac Surg 22: 679-689, 2011.

4. Schrumpf MA, Lee AT and Weiland AJ: Foreign-body reaction and osteolysis induced by an intraosseous poly-L-lactic Acid suture anchor in the wrist: Case report. J Hand Surg Am 36: 1769-1773, 2011

5. Kim SH, Oh JH, Lee OS, Lee HR and Hargens AR: Postoperative imaging of bioabsorbable anchors in rotator cuff repair. Am J Sports Med 42: 552-557, 2014.

6. Fu D, Xiao B, Yang S and Li J: Open reduction and bioabsorbable pin fixation for late presenting irreducible supracondylar humeral fracture in children. Int Orthop 35: 725-730, 2011.

7. Waris E, Ashammakhi N, Raatikainen T, Törmälä P, Santavirta S and Konttinen YT: Self-reinforced bioabsorbable versus metallic fixation systems for metacarpal and phalangeal fractures: A biomechanical study. J Hand Surg Am 27: 902-909, 2002.

8. Waris E, Ashammakhi N, Kaarela O, Raatikainen $\mathrm{T}$ and Vasenius J: Use of bioabsorbable osteofixation devices in the hand. J Hand Surg Br 29: 590-598, 2004.

9. Claes L, Recknagel S and Ignatius A: Fracture healing under healthy and inflammatory conditions. Nat Rev Rheumatol 8: 133-143, 2012

10. Gerstenfeld LC, Cullinane DM, Barnes GL, Graves DT and Einhorn TA: Fracture healing as a post-natal developmental process: Molecular, spatial, and temporal aspects of its regulation. J Cell Biochem 88: 873-884, 2003.

11. Rennert J, Coffman JA, Mushegian AR and Robertson AJ: The evolution of Runx genes I. A comparative study of sequences from phylogenetically diverse model organisms. BMC Evol Biol 3: 4, 2003.

12. Bustamante M, Nogués X, Agueda L, Jurado S, Wesselius A, Cáceres E, Carreras R, Ciria M, Mellibovsky L, Balcells S, et al: Promoter $2-1025$ T/C polymorphism in the RUNX2 gene is associated with femoral neck bmd in Spanish postmenopausal women. Calcif Tissue Int 81: 327-332, 2007.

13. Qin X, Jiang Q, Matsuo Y, Kawane T, Komori H, Moriishi T, Taniuchi I, Ito K, Kawai Y, Rokutanda S, et al: Cbfb regulates bone development by stabilizing Runx family proteins. J Bone Miner Res 30: 706-714, 2015.

14. Wu M, Li C, Zhu G, Wang Y, Jules J, Lu Y, McConnell M, Wang YJ, Shao JZ, Li YP and Chen W: Deletion of core-binding factor $\beta(\mathrm{Cbf} \beta)$ in mesenchymal progenitor cells provides new insights into $\mathrm{Cbf} \beta /$ Runxs complex function in cartilage and bone development. Bone 65: 49-59, 2014.

15. Xu H, Duan J, Ning D, Li J, Liu R, Yang R, Jiang JX and Shang P. Role of Wnt signaling in fracture healing. BMB Rep 47: 666-672, 2014.
16. Eastman Q and Grosschedl R: Regulation of LEF-1/TCF transcription factors by Wnt and other signals. Curr Opin Cell Biol 11: 233-240, 1999.

17. Zhong N, Gersch RP and Hadjiargyrou M: Wnt signaling activation during bone regeneration and the role of Dishevelled in chondrocyte proliferation and differentiation. Bone 39: 5-16, 2006.

18. Chen Y, Whetstone HC, Lin AC, Nadesan P, Wei Q, Poon R and Alman BA: Beta-catenin signaling plays a disparate role in different phases of fracture repair: Implications for therapy to improve bone healing. PLoS Med 4: e249, 2007.

19. Gaur T, Lengner CJ, Hovhannisyan H, Bhat RA, Bodine PV, Komm BS, Javed A, van Wijnen AJ, Stein JL, Stein GS and Lian JB: Canonical WNT signaling promotes osteogenesis by directly stimulating Runx2 gene expression. J Biol Chem 280: 33132-33140, 2005.

20. Rosier RN, O'Keefe RJ and Hicks DG: The potential role of transforming growth factor beta in fracture healing. Clin Orthop Relat Res (Suppl 355): S294-S300, 1998.

21. Livak KJ and Schmittgen TD: Analysis of relative gene expression data using real-time quantitative PCR and the 2(-Delta Delta C(T)) Method. Methods 25: 402-408, 2001.

22. Mabilleau G, Mieczkowska A, Libouban H, Simon Y, Audran M and Chappard D: Comparison between quantitative X-ray imaging, dual energy X-ray absorptiometry and microCT in the assessment of bone mineral density in disuse-induced bone loss. J Musculoskelet Neuronal Interact 15: 42-52, 2015.

23. Yonden Z, Aydin M, Alcin E, Kelestemur MH, Kutlu S and Yilmaz B: Effects of letrozole on bone biomarkers and femur fracture in female rats. J Physiol Biochem 65: 267-275, 2009.

24. Fahrleitner-Pammer A, Dobnig H, Piswanger-Soelkner C, Bonelli C, Dimai HP, Leb G and Obermayer-Pietsch B: Osteoprotegerin serum levels in women: Correlation with age, bone mass, bone turnover and fracture status. Wien Klin Wochenschr 115: 291-297, 2003.

25. Yee CS, Xie L, Hatsell S, Hum N, Murugesh D, Economides AN, Loots GG and Collette NM: Sclerostin antibody treatment improves fracture outcomes in a Type I diabetic mouse model. Bone 82: 122-134, 2016

26. Komori T, Yagi H, Nomura S, Yamaguchi A, Sasaki K, Deguchi K, Shimizu Y, Bronson RT, Gao YH, Inada M, et al: Targeted disruption of Cbfa1 results in a complete lack of bone formation owing to maturational arrest of osteoblasts. Cell 89: 755-764, 1997.

27. Inada M, Yasui T, Nomura S, Miyake S, Deguchi K, Himeno M, Sato M, Yamagiwa H, Kimura T, Yasui N, et al: Maturational disturbance of chondrocytes in Cbfal-deficient mice. Dev Dyn 214: 279-290, 1999.

28. Ueta C, Iwamoto M, Kanatani N, Yoshida C, Liu Y, Enomoto-Iwamoto M, Ohmori T, Enomoto H, Nakata K, Takada K, et al: Skeletal malformations caused by overexpression of Cbfal or its dominant negative form in chondrocytes. J Cell Biol 153: 87-100, 2001.

29. Zhang Y, Hassan MQ, Montecino M, Hawse JR, Spelsberg TC, Stein JL, Lian JB, van Wijnen AJ and Stein GS: Synergistic regulation of the Runx $2 \mathrm{P} 1$ promoter in mesenchymal cells by a conserved HLH box and purine-rich elements (GAY motifs). FASEB J 22 (Suppl 1): S782.717, 2008.

30. Doecke JD, Day CJ, Stephens AS, Carter SL, van Daal A, Kotowicz MA, Nicholson GC and Morrison NA: Association of functionally different RUNX2 P2 promoter alleles with BMD. J Bone Miner Res 21: 265-273, 2006.

31. Harada H, Tagashira S, Fujiwara M, Ogawa S, Katsumata T, Yamaguchi A, Komori T and Nakatsuka M: Cbfa1 isoforms exert functional differences in osteoblast differentiation. J Biol Chem 274: 6972-6978, 1999.

32. Liu JC, Lengner CJ, Gaur T, Lou Y, Hussain S, Jones MD, Borodic B, Colby JL, Steinman HA, van Wijnen AJ, et al: Runx2 protein expression utilizes the Runx2 P1 promoter to establish osteoprogenitor cell number for normal bone formation. J Biol Chem 286: 30057-30070, 2011.

33. Tamiya H, Ikeda T, Jeong JH, Saito T, Yano F, Jung YK, Ohba S, Kawaguchi H, Chung UI and Choi JY: Analysis of the Runx2 promoter in osseous and non-osseous cells and identification of HIF2A as a potent transcription activator. Gene 416: 53-60, 2008.

34. Hirata M, Kugimiya F, Fukai A, Saito T, Yano F, Ikeda T, Nakamura K, Chung UI and Kawaguchi H: 060 Molecular network on the C/Ebp-Beta axis including Runx2, Mmp13, and Hif2a controls osteoarthritis development. Osteoarthritis Cartilage 18 (Suppl 2): S34, 2010. 
35. Heidemann W, Jeschkeit S, Ruffieux K, Fischer JH, Wagner M, Krüger G, Wintermantel E and Gerlach KL: Degradation of poly (D,L)lactide implants with or without addition of calciumphosphates in vivo. Biomaterials 22: 2371-2381, 2001.

36. Jansen J, Koopmans SA, Los LI, van der Worp RJ, Podt JG, Hooymans JM, Feijen J and Grijpma DW: Intraocular degradation behavior of crosslinked and linear poly(trimethylene carbonate) and poly(D,L-lactic acid). Biomaterials 32: 4994-5002, 2011.

37. Carroll SH and Ravid K: Differentiation of mesenchymal stem cells to osteoblasts and chondrocytes: A focus on adenosine receptors. Expert Rev Mol Med 15: e1, 2013.
38. Kim JH, Liu X, Wang J, Chen X, Zhang H, Kim SH, Cui J, Li R, Zhang W, Kong Y, et al: Wnt signaling in bone formation and its therapeutic potential for bone diseases. Ther Adv Musculoskelet Dis 5: 13-31, 2013

39. Monroe DG, McGee-Lawrence ME, Oursler MJ and Westendorf JJ: Update on Wnt signaling in bone cell biology and bone disease. Gene 492: 1-18, 2012.

40. Simon JA, Ricci JL and Di Cesare PE: Bioresorbable fracture fixation in orthopedics: A comprehensive review. Part I. Basic science and preclinical studies. Am J Orthop (Belle Mead NJ) 26: 665-671, 1997. 